\title{
Effect of Lesioning the Ventral Tegmental Area on Food Intake, Water Intake, Body Weight and Alcohol Consumption in Wistar Albino Rats
}

\author{
Santosh K MAYANNAVAR ${ }^{1}$, Murlimanju, B.V. ${ }^{2}$, Gaurav Sharma B ${ }^{3}$, Ganaraja BOLUMBU ${ }^{4}$ \\ ${ }^{1}$ Shri Vinoba Bhave Civil Hospital, Department of Physiology, Silvassa, Dadara and Nagar Haveli (UT), India \\ ${ }^{2}$ Kasturba Medical College, Mangalore, (Manipal Academy of Higher Education, Manipal) Department of Anatomy, Karnataka, India \\ ${ }^{3}$ Countess of Chester NHS Hospital, Department of Orthopaedics, Chester, Cheshire, CH2 1UL, UK \\ ${ }^{4}$ Kasturba Medical College, Mangalore, (Manipal Academy of Higher Education, Manipal) Department of Physiology, Karnataka, India
}

Corresponding author: Ganaraja BOLUMBU ganaraja.b@manipal.edu; ganaraj.b@gmail.com

\section{ABSTRACT}

AIM: To study the effect of lesion of the ventral tegmental area (VTA) on food intake, water intake, alcohol consumption, and alcohol preference in the post-lesion period.

MATERIAL and METHODS: A group of 38 young adult healthy male Wistar albino rats was included in this experiment. The groups of rats were subjected to the bilateral electrolytic lesion of VTA, and the effect on water intake, food intake, alcohol consumption, and alcohol preference in the post-lesion period was analyzed. A sham lesioned control group was maintained for all experimental groups.

RESULTS: There was no change observed in food intake and the body weight in lesioned animals ( $p>0.05)$. It was found that increased water intake was observed if only water was given. There was also increased alcohol intake when alcohol was only provided $(p<0.05)$. However, two bottle-free choice tests for alcohol preference revealed that the rats preferred to consume water and not alcohol when the choice was given. There was an increase in the total volume consumed $(p<0.05)$.

CONCLUSION: There was an increase in water intake and no notable changes in the food intake and body weight following the lesion. When offered alone, alcohol consumption was raised, but when a choice of alcohol and water was given in a two-bottle freechoice test, rats preferred to drink water. The present study suggests that VTA lesions may be associated with increased thirst and high fluid consumption, which may have implications in water balance.

KEYWORDS: Alcohol consumption, Ventral tegmental area, Water intake

\section{INTRODUCTION}

$\mathrm{V}$ Tentral tegmental area (VTA) is a collection of neuronal cells on the floor of mesencephalon. VTA is considered as the source of mesolimbic dopaminergic pathways (13). The role of this part of the brain stem in the direct regulation of ingestion related activities, particularly the quantitative and qualitative aspects of the food consumed is largely sketchy. Though this part of the brain has been known to have extensive connections with the basal brain, its role in the feeding behaviors and alcohol intake is still not understood. There are very few articles available in the literature, which address these issues. Few reports revealed a vast number of neuronal connections between the VTA and hypothalamic areas directly related to the feeding activities, viz. The lateral hypothalamus (LH) and other forebrain areas. The dopaminergic circuit arising from VTA has been an essential component of the reward mechanism. Neuronal connections of VTA arborize all over the forebrain, including the nucleus accumbens and frontal lobe of the cerebral cortex,
Santosh K MAYANNAVAR (D) : 0000-0002-2838-8014 Murlimanju B.V $\quad$ (D) : 0000-0003-1248-8296
Gaurav Sharma B (D) : 0000-0002-3463-6803

Ganaraja BOLUMBU (D) : 0000-0002-8023-6851 
dopamine being the primary neurotransmitter (1, 9). Dopamine is also a vital neurotransmitter, which is essential for the motor activities concerned with the feeding. It was reported that the mice that lack the enzyme tyrosine hydroxylase were unable to feed and died in about three weeks (16). It was observed that treating these animals with dopamine or L-dopa improved motor activity and feeding, which increased their survival. This observation suggested that there is a close link between dopamine and feeding behavior. The dopaminergic neurons of VTA in addiction to methamphetamine involves the potassium current through the G-protein-gated inwardly rectifying potassium (GIRK3) channels (10).

The lateral hypothalamic area, the primary center for the control of feeding behaviour, has been reportedly well connected with the VTA. Thus the VTA dopaminergic system influences the motivated behaviours apart from the addiction, food, and water intake (7). The present study aimed to evaluate the effect of electrolytically lesioned VTA region and the quantity of food intake, water intake, and body weight. Further, to assess if the VTA has any specific role in alcohol consumption, the objective was to study the measurement of alcohol intake.

\section{MATERIAL and METHODS}

A group of 38 young adult healthy male Wistar albino rats weighing about $260 \pm 25 \mathrm{~g}$ weight were chosen for this experiment. Ethical clearance was obtained from the animal ethics committee of the institution before the beginning of the experimental procedure. All measures were meticulously followed to treat the animals with care. The rats were housed in a cool and dry room, individual cages with paddy husk bedding, and provided with ad libitum food and potable water in normal day-night cycle. They were maintained for a week before the intervention started.

\section{Grouping}

The rats were divided into 6 groups namely:

Group 1 (Control, Sham lesioned) - water; Group 1a (Experimental) - water $(\mathrm{n}=6$ each, one animal died after surgery)

Group 2 (Control) - alcohol; Group 2a (Experimental) - alcohol $(n=6$ each, one animal died after surgery)

Group 3 (Control) -2 bottle; Group 3a (Experimental) - 2 bottle (water+alcohol).

A total of 38 animals $(36+2$ models died) were used for the present research.

\section{Pre-lesion measurements}

The animals were weighed, and their pretreatment weight was noted in grams. They were provided with a known quantity of water in the nostled drinking bottle, which was replenished every morning after recording daily consumption. Similarly, $10 \%$ alcohol was prepared from $100 \%$ ethanol (Hayman Ltd) by adding distilled water and was given in the drinking bottle (in alcohol group). Rat feed pellets were also weighed and provided every day, the leftover was weighed, and the consumption was noted after taking into account the wastage. All measurements were carried out between $9 \mathrm{am}$, and $11 \mathrm{am}$. Their body weight was monitored every week.

\section{Surgical procedure}

Ketamine (60 mg/kg body weight) and xylazine $(6 \mathrm{mg} / \mathrm{kg}$ body weight) were injected intraperitoneally to induce anesthesia. The head was shaved, and bregma was marked on the skull. Stereotaxic coordinates for VTA was $(A P=-6.7 \mathrm{~mm}$; $L= \pm 0.5$ $\mathrm{mm} ; \mathrm{V}=8.1 \mathrm{~mm}$ ) were marked (Figure 1 ) according to Paxinos \& Watson rat stereotaxic atlas (12). Lesions were produced by standard procedure (6). Burr holes were made bilaterally. Stainless steel electrode, which was insulated except for the $0.5 \mathrm{~mm}$ at the tip, was lowered using stereotaxic apparatus. Two $\mathrm{mA}$ current was passed into each side VTA area for $20 \mathrm{sec}$, and the electrode was withdrawn using stimulator assembly (Grass, USA). Following this, the burr holes were sealed using the dental cement, and the skin was sutured. Antiseptic was applied to the wound, and the rats were injected penicillin (1 lakh units) single dose, to prevent infection. The rats were transferred to the home cage and allowed to recover for two days. Following recovery, the daily measurement of parameters was carried out for two weeks. For each group, a sham lesioned control group was parallelly maintained for ruling out the variations in consumption due to surgical damage to structures. These animals received all the treatments and surgical procedures in the same way the experimental group, except the current, was not passed, and hence there is no electrolytic lesion.

\section{Post-lesion measurements}

Food intake, water intake, alcohol intake were recorded meticulously before (prelesion) and followed surgical recovery (post-lesion) mentioned above for two weeks. Weekly changes in body weight were also recorded.

\section{Alcohol preference test}

The preference test was performed using the 'two-bottle free choice' situation, as explained in our previous study (5). The rats were offered two bottles filled with $10 \%$ ethanol in one bottle and potable water in the other bottle. The access was given for 24 hours on all days of observation. The position of the bottles was randomly changed. The volume of intake from each bottle was noted, and the total intake was also recorded. The consumption was measured for the prelesion period and two weeks following recovery from surgery.

\section{Euthanasia and brain dissection}

At the end of 2 weeks of observation and recording data, the rats were sacrificed by a lethal dose of ether anesthesia and transfused with formal saline (intracardiac). The brain was quickly dissected out and washed in saline and stored for histological examination to confirm lesion accuracy. Fivemicron slices were cut from the wax blocks and stained to observe the correctness of lesion.

The mean consumption of fluids, namely alcohol (10\%) or water as the case may be in each group, was measured. These mean values were calculated for one week before the 


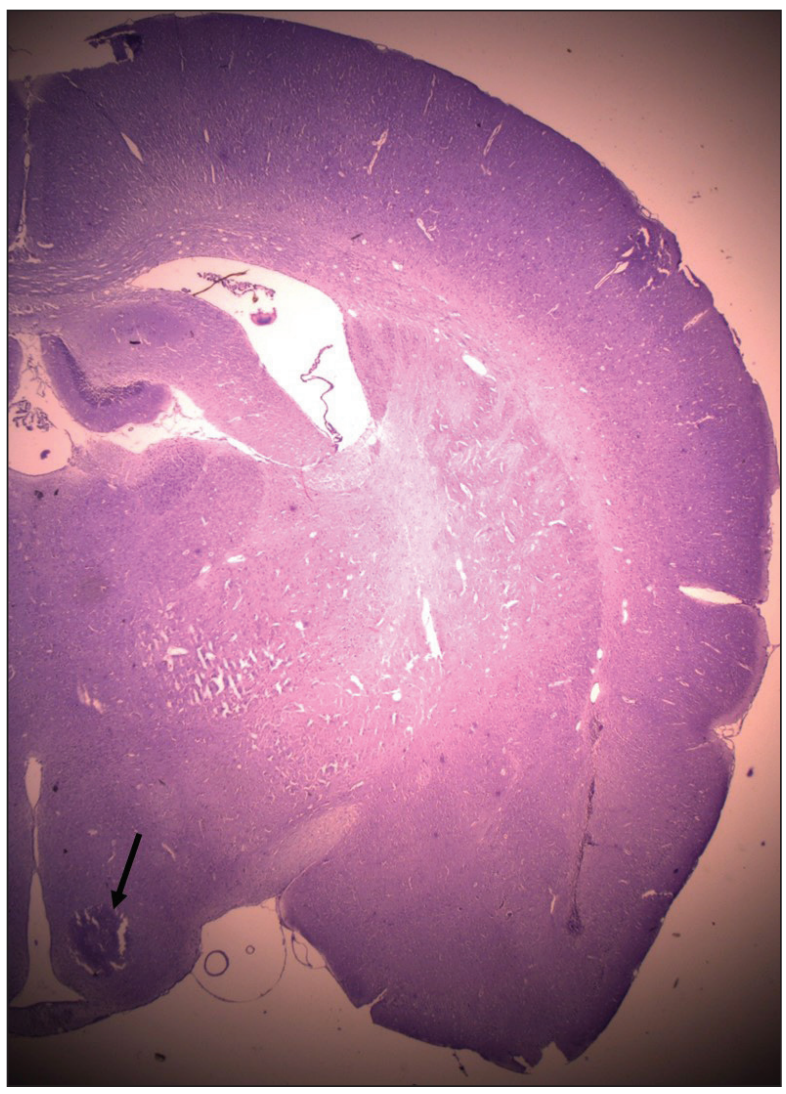

Figure 1: Marking of the site of lesion in VTA $(\downarrow)$ was performed as per the stereotaxic atlas (Paxinos \& Watson). lesion (prelesion) and two weeks following the lesion (postlesion week one and week 2) and tabulated. The values were compared, and the differences were noted and statistically compared using the online software (8). The non-parametric Wilcoxon's signed-rank sum test and Student's 't' test were applied for the statistical analysis. The statistical significance was considered if the ' $p$ ' value is less than $0.05(p<0.05)$.

\section{RESULTS}

Table I represents the alcohol (ethanol 10\%) intake, daily food intake, and weekly changes in the body weight in Sham lesioned control, and bilateral VTA lesioned alcohol groups of rats.

Alcohol intake (Table I): It was observed that, the comparison was statistically significant in the pre lesion Vs post lesion 1, alcohol intake groups (Wilcoxon signed rank sum test; $z=$ -2.2014; $w=0 ; n=5 ; p<0.05)$. Pre lesion Vs Post lesion week 2 comparison was also significant $(z=-2.2014 ; w=1 ; n=6 ; p<$ 0.05).

Food intake (Table I): The comparison was not significant for the food intake between prelesion Vs. Week 1 and week 2.

Body weight (Table I): The comparison was statistically significant for the bodyweight, pre lesion Vs. week $1(\mathrm{~W}=1$; $\mathrm{n}=6 ; \mathrm{p}<0.05$ ). But this comparison was not significant (pre lesion Vs. week 2; p>0.05). The analysis of the same data by applying the Student's 't' test was not significant (pre lesion Vs. post-lesion week 1 and 2).

Table I: Intake of Alcohol (ethanol 10\%), Food and Weekly Changes in Body Weight in Sham Lesioned Control and Bilateral VTA Lesioned Groups of Rats

\begin{tabular}{|c|c|c|c|c|c|c|c|c|c|c|}
\hline & \multicolumn{5}{|c|}{ Alcohol ml/day } & \multirow{2}{*}{$\begin{array}{l}\text { Food g/day } \\
\text { post lesion p } \\
\text { wk } 1\end{array}$} & \multicolumn{4}{|c|}{ Body weight weekly (g) } \\
\hline & $\begin{array}{c}\text { rat } \\
\text { number }\end{array}$ & prelesion & $\begin{array}{c}\text { post lesion } \\
\text { wk 1* }\end{array}$ & $\begin{array}{c}\text { post lesion } \\
\text { wk 2* }\end{array}$ & prelesion & & $\begin{array}{l}\text { ost lesion } \\
\text { wk } 2\end{array}$ & prelesion & $\begin{array}{c}\text { post lesic } \\
\text { wk } 1\end{array}$ & $\begin{array}{l}\text { wk } 2^{*} \\
\text { wost lesion }\end{array}$ \\
\hline \multirow{6}{*}{$\begin{array}{l}\text { Control } \\
\text { rats }(n=6)\end{array}$} & 1 & 18.5 & 18 & 17.8 & 13.5 & 14.2 & 12.8 & 265 & 268 & 271 \\
\hline & 2 & 17.8 & 19 & 16.5 & 13 & 13.5 & 13.2 & 250 & 252 & 260 \\
\hline & 3 & 20 & 19.3 & 18.7 & 12 & 13.2 & 14 & 292 & 284 & 288 \\
\hline & 4 & 16.9 & 18 & 18.4 & 12.8 & 12.7 & 13.2 & 260 & 164 & 270 \\
\hline & 5 & 14.7 & 15.9 & 16.8 & 14 & 13.5 & 12.9 & 274 & 276 & 281 \\
\hline & 6 & 17 & 17.2 & 17 & 13.4 & 14 & 13.2 & 234 & 238 & 242 \\
\hline \multirow{6}{*}{$\begin{array}{l}\text { VTA } \\
\text { lesioned } \\
(n=6)\end{array}$} & 1 & 23.8 & 21 & 22 & 12.71 & 13.42 & 13 & 276 & 298 & 280 \\
\hline & 2 & 22.8 & 20 & 21.7 & 12.8 & 13.32 & 13.1 & 252 & 260 & 265 \\
\hline & 3 & 20.5 & 20 & 20.3 & 12.85 & 12.28 & 12 & 268 & 293 & 313 \\
\hline & 4 & 18.85 & 13 & 15 & 12.28 & 11.5 & 12.5 & 270 & 295 & 278 \\
\hline & 5 & 21 & 19.8 & 20 & 12.28 & 11.5 & 11.8 & 253 & 262 & 290 \\
\hline & 6 & 18.8 & 13.8 & 16 & 10.28 & 10.85 & 11 & 248 & 244 & 225 \\
\hline
\end{tabular}

Legend: Values mean of 7 days. Ethanol consumption (ml/day) increased significantly in the post-lesion week $1 \& 2$ ( $p<0.05)$. There was no significant change in the food intake. There was moderate increase in the body weight. ${ }^{*} p<0.05$. 
Table II represents the water intake, and food intake in Sham lesioned control and bilateral VTA lesioned groups.

Water intake (Table II): The comparison of water intake in groups pre lesion Vs. post-lesion week 1, was statistically significant $(\mathrm{z}=-2.2014 ; \mathrm{W}=0 ; \mathrm{n}=6 ; \mathrm{p}<0.05)$. The comparison was also statistically significant $(p<0.05)$ for the comparison between pre lesion Vs post-lesion, week 2 ( $z=-2.2014$; $W=0 ; n=6 ; p<0.05)$. The analysis of the same data by applying Student's 't' test was also significant $(\mathrm{t}=-2.03666, \mathrm{p}=0.034516$. The result is significant at $p<0.05)$.
Table 3 indicates the results of the two-bottle free choice test. The intake of water, ethanol (10\%), and total fluid intake were compared in control and VTA lesioned rats. The mean values for seven days were taken.

Water intake (Table III): The comparison of water intake was statistically significant $(p<0.05)$ between the groups. prelesion Vs. post-lesion week $1(z=-2.2014 ; W=0 ; n=6 ; p<0.05)$. The Student's 't' test was also applied to compare pre- lesion Vs. post-lesion week $2(t=-2.64786 ; p=0.012201)$. The result

Table II: Intake of Water and Food in Control and Bilateral VTA Lesion Lesioned Groups of Rats

\begin{tabular}{|c|c|c|c|c|c|c|c|}
\hline & \multirow[b]{2}{*}{ rat number } & \multicolumn{3}{|c|}{ Water ml/day } & \multicolumn{3}{|c|}{ Food g/day } \\
\hline & & prelesion & $\begin{array}{c}\text { post lesion } \\
\text { wk 1* }\end{array}$ & $\begin{array}{c}\text { post lesion } \\
\text { wk 2* }\end{array}$ & prelesion & $\begin{array}{c}\text { post lesion } \\
\text { wk } 1\end{array}$ & $\begin{array}{c}\text { post lesion } \\
\text { wk } 2\end{array}$ \\
\hline \multirow{4}{*}{$\begin{array}{l}\text { Control rats } \\
(n=6)\end{array}$} & 2 & 18.8 & 18.6 & 18.5 & 15 & 14.5 & 14.6 \\
\hline & 3 & 17.3 & 19.1 & 18.8 & 14.5 & 13.9 & 14.3 \\
\hline & 5 & 16.5 & 15.9 & 16.8 & 14.5 & 13.8 & 13.9 \\
\hline & 6 & 17 & 16.8 & 17.1 & 14.4 & 13.4 & 13.2 \\
\hline \multirow{4}{*}{$\begin{array}{l}\text { VTA lesioned } \\
(n=6)\end{array}$} & 1 & 21.3 & 23.2 & 26 & 12.7 & 13.4 & 13.9 \\
\hline & 2 & 20.1 & 23.4 & 23.7 & 13.8 & 14.2 & 13.4 \\
\hline & 3 & 19 & 21 & 20.3 & 12.8 & 11.8 & 12.5 \\
\hline & 6 & 18.8 & 20.2 & 22.8 & 13.8 & 13.8 & 13.2 \\
\hline
\end{tabular}

Legend: Values mean of 7 days. Water consumption (ml/day) increased significantly in the post-lesion week 1 \& 2 ( $p<0.05$ ). There was no significant change in the food intake ${ }^{*} p<0.05$.

Table III: Two Bottle Free Choice Test. (Intake of Ethanol (10\%), Water and Total Fluid) in Control and VTA Lesioned Rats

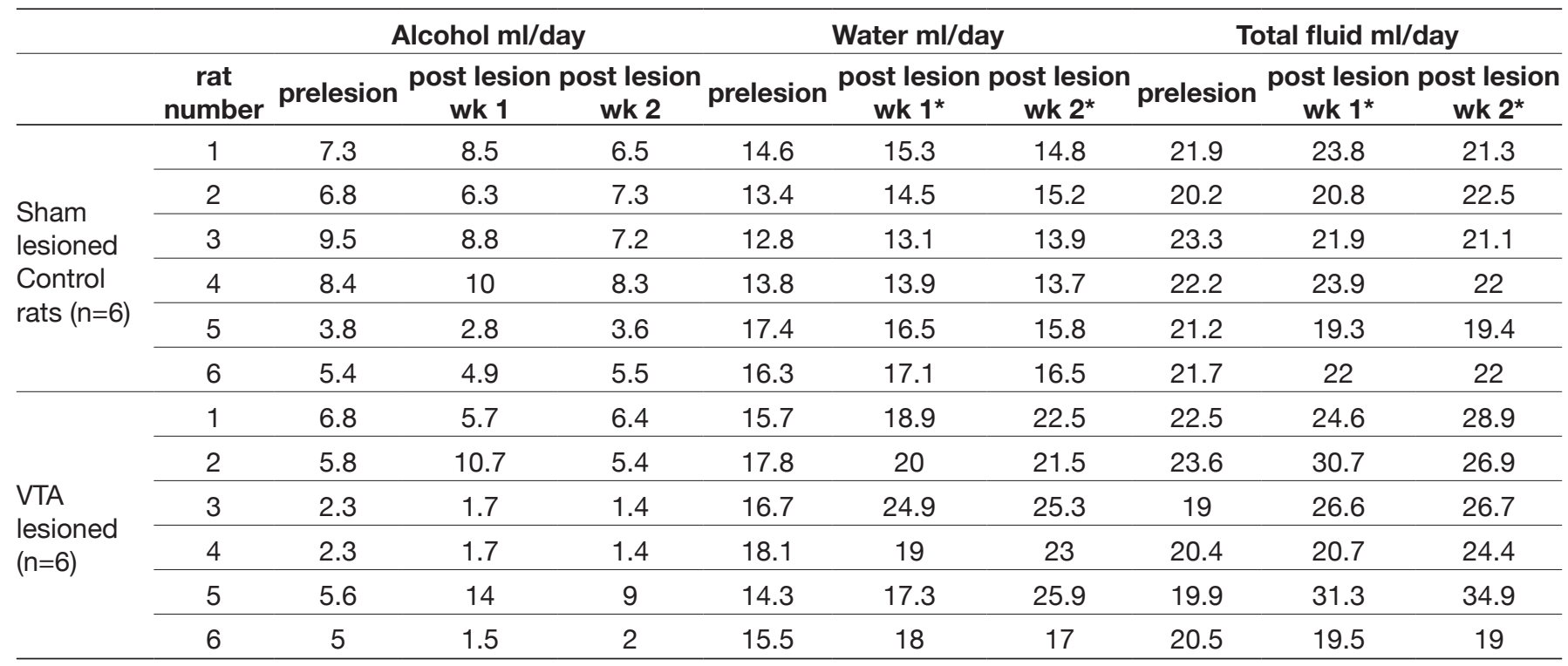

Legend: Values mean for the 7 days. Two bottle free choice test for preference, Water consumption (ml/day) increased significantly in the postlesion week 1 \& 2 . No significant change in alcohol intake. Significant increase in total fluid intake. ${ }^{*} p<0.05$. 
is significant at $\mathrm{p}<0.05$. Ethanol $(10 \%)$ consumption did not show any statistically significant difference.

Total fluid intake (Table III): The comparison of total fluid intake was statistically significant $(p<0.05)$ between the groups pre lesion Vs post-lesion week 1 ( $z=-2.2014 ; \mathrm{W}=0$; $n=6 ; p<0.05)$. The same comparison was also significant when Student's 't'-test was applied ( $t=-2.14837 ; p=0.028611)$. This was statistically significant as $p<0.05$. The comparison between pre lesion and post-lesion week 2 was also significant with the Student's 't' test at $\mathrm{p}<0.05(\mathrm{t}=-2.58865 ; \mathrm{p}=0.013506)$.

The present study observed that the food intake did not show a significant difference ( $p>0.05)$ among the groups, prelesion, and post-lesion period in week one and week 2 . There were also no detectable changes in the body weight in pre and post-lesion periods. However, it was found that increased water intake was observed if only water was given. There is also increased alcohol intake when alcohol was only provided $(p<0.05)$. However, a two-bottle free-choice test for alcohol preference revealed that the rats preferred to consume water and not alcohol when the choice was given. There was an increase in the total volume consumed $(p<0.05)$.

\section{DISCUSSION}

Pratt et al. (15) demonstrated that a 5HT3 receptor-mediated histaminergic system was critical in VTA mediated food intake. $5 \mathrm{HT} 3$ receptor agonists in VTA decreased food intake while it increased food intake when applied to Nucleus accumbens. Glutamatergic inputs to VTA from the prefrontal area could be a factor that regulated the feeding activities, as evidenced by the use of NMDA receptor blocker memantine into rat VTA (11). Serotonergic receptor manipulation in VTA revealed that $5 \mathrm{HT} 1 \mathrm{~b}$ receptor antagonists decreased the intake of palatable food in food-deprived rats (14). In the present study, we investigated VTA's role in influencing alcohol consumption, which may have a role in alcohol addiction. We also tested the intake of food and water and changes in the body weight, indicating the general effects in ingestive behaviour. The lesion of VTA could alter the behaviors, which will indicate its regulatory role in these behaviours. The results suggested an increase in alcohol consumption in the lesioned animals when alcohol alone was provided for drinking. However, we tested the alcohol preference to confirm whether they prefer alcohol over water in the post-lesion period. It was found that there was an increase in the intake of water, and there was an increase in total fluid intake. But there was no increment in the consumption of $10 \%$ alcohol. From this result, it may be inferred that in the current experiment, there is evidence to state that VTA lesion produced an increased intake of water. Still, it does not cause a preference for alcohol intake, and probably this indicated an increased thirst in such animals.

Motivational behaviour for feeding, addiction, and motor activity requires the stimulation of dopaminergic neurons of VTA (2). It was found that the dopaminergic activation of VTA has increased the sucrose consumption, but substantia nigra failed to produce such a response. This observation highlighted the VTA dopaminergic system in motivational behaviour. The dopaminergic fibers arising from the VTA to basal forebrain and forebrain structures affected the pattern of eating behaviour (3). Though the authors did not observe the decrease in the total food intake for the day, they found that the meal size decreased considerably. At the same time, the animals consumed in short bouts could be attributed to the increased locomotor activity. In the present study, we did not find any changes in the quantity of food consumption. Since there was no increase in food intake, there was no increase in body weight. These findings are in agreement with the reports from previous authors. In other reports, orexinergic inputs into VTA acting through OX1R \& OX2R receptors reportedly increased the intake of palatable food when tested with sucrose solution (17). Our study did not use a taste solution, but rewarding alcohol was used. There was no preference for alcohol.

Other reports also stated that the serotonergic system, more specifically $5 \mathrm{HT} 1 \mathrm{~b}$ receptors, were implicated in sweet taste preference (14). The above two observations strengthen the relationship between the LH and VTA, which suggests that VTA projected to the hypothalamus via the dopaminergic pathway. On the other hand, LH communicated with midbrain through the orexinergic pathway. The dopaminergic system from VTA and orexinergic inputs to VTA from LH may have a role in the feeding behaviour, and substance addiction and abuse (4). The strong relationship of VTA with those centres concerned with food and water intake in the hypothalamus suggested VTA's influence in ingestive behaviour, which now appears to be related to the palatable food and fluid. From our study, we conclude that VTA inputs to the hypothalamus are essential for the thirst and drinking, which is primarily concerned with water balance. Lesioning of VTA did not lead to changes in body weight or food intake in our study.

\section{CONCLUSION}

The present study performed a bilateral lesion of VTA and studied the water intake, alcohol intake, food intake, and body weight changes in Wistar albino rats. There was increased water intake, and no notable changes in the food intake and body weight in the lesioned animals. Alcohol consumption, when offered alone, was raised. To detect the alcohol preference, a specific test, 'two-bottle free choice', was carried out by providing two drinking bottles simultaneously. When a choice of alcohol and water was provided in this test, they preferred water, which suggested VTA's role in water balance and thirst.

\section{REFERENCES}

1. Barrot M, Sesack SR, Georges F, Pistis M, Hong S, Jhou TC: Braking dopamine systems: A new GABA master structure for mesolimbic and nigrostriatal functions. J Neurosci 32:1409414101, 2012

2. Boekhoudt L, Roelofs TJM, de Jong JW, de Leeuw AE, Luijendijk MCM, Wolterink-Donselaar IG, van der Plasse G, Adan RAH: Does activation of midbrain dopamine neurons promote or reduce feeding? Int J Obes (Lond) 41:1131-1140, 2017 
3. Boekhoudt L, Wijbrans EC, Man J, Luijendijk MCM, De Jong $H$, van der Plasse $G$, Vanderschuren $L$, Adan $R$ : Enhancing excitability of dopamine neurons promotes motivational behaviour through increased action initiation. Eur Neuropsychopharmacol 28:171-184, 2018

4. Farahimanesh $S$, Zarrabian $S$, Haghparast A: Role of orexin receptors in the ventral tegmental area on acquisition and expression of morphine-induced conditioned place preference in the rats. Neuropeptides 66:45-51, 2017

5. Ganaraja B, Jeganathan PS: Effect of basolateral amygdala \& ventromedial hypothalamic lesions on ingestion \& taste preference in rat. Indian J Med Res 112:65-70, 2000

6. Ganaraja B, Jeganathan PS: Involvement of basolateral nucleus \& central nucleus of amygdala in the regulation of ingestive behaviour in rat. Indian J Med Res 108:98-103, 1998

7. Hurley SW, Johnson AK: The role of the lateral hypothalamus and orexin in ingestive behavior: A model for the translation of past experience and sensed deficits into motivated behaviors. Front Syst Neurosci 8:216, 2014

8. Internet Source: https://www.socscistatistics.com/tests/ signedranks/default2.aspx; Accessed on March 25, 2020

9. Malenka RC, Nestler EJ, Hyman SE: Widely projecting systems: Monoamines, acetylcholine, and orexin. In: Sydor A, Brown RY (ed), Molecular Neuropharmacology: A Foundation for Clinical Neuroscience, $2^{\text {nd }}$ ed, New York: McGraw-Hill Medical, 2009:147-148, 154-157

10. Munoz MB, Padgett CL, Rifkin R, Terunuma M, Wickman K, Contet C, Moss SJ, Slesinger PA: A role for the GIRK3 subunit in methamphetamine-induced attenuation of GABAB receptor-activated GIRK currents in VTA dopamine neurons. J Neurosci 36:3106-3114, 2016
11. Nasihatkon ZS, Khosravi M, Bourbour Z, Sahraei H, Ranjbaran M, Hassantash SM, Sahraei M, Baghlani K: Inhibitory effect of NMDA receptors in the ventral tegmental area on hormonal and eating behavior responses to stress in rats. Behav Neurol 2014:294149, 2014

12. Paxinos G, Watson C: Rat Brain in Stereotaxic Coordinates, $5^{\text {th }}$ ed, Amsterdam: Elsevier Academic Press, 1998

13. Phillipson OT: Afferent projections to the ventral tegmental area of Tsai and interfascicular nucleus: A horseradish peroxidase study in the rat. J Comp Neurol 187:117-143, 1979

14. Pratt WE, Clissold KA, Lin P, Cain AE, Ciesinski AF, Hopkins TR, llesanmi AO, Kelly EA, Pierce-Messick Z, Powell DS, Rosner IA: A systematic investigation of the differential roles for ventral tegmentum serotonin 1- and 2-type receptors on food intake in the rat. Brain Res 1648:54-68, 2016

15. Pratt WE, Lin P, Pierce-Messick Z, llesanmi AO, Clissold KA: Contrasting effects of 5-HT3 receptor stimulation of the nucleus accumbens or ventral tegmentum on food intake in the rat. Behav Brain Res 323:15-23, 2017

16. Szczypka MS, Rainey MA, Kim DS, Alaynick WA, Marck BT, Matsumoto AM, Palmiter RD: Feeding behavior in dopaminedeficient mice. Proc Natl Acad Sci USA 96:12138-12143, 1999

17. Terrill SJ, Hyde KM, Kay KE, Greene HE, Maske CB, Knierim AE, Davis JF, Williams DL: Ventral tegmental area orexin 1 receptors promote palatable food intake and oppose postingestive negative feedback. Am J Physiol Regul Integr Comp Physiol 311:R592-R599, 2016 